\title{
Amnesia: um Objeto de Aprendizagem para o Ensino de Hierarquia de Memória
}

\author{
Fernando Tiosso, Sarita Mazzini Bruschi, Paulo Sergio Lopes de Souza, Ellen \\ Francine Barbosa \\ Instituto de Ciências Matemáticas e de Computação - ICMC/USP \\ Caixa Postal 668 - 13.560-970 - São Carlos - SP - Brasil \\ \{ftiosso, sarita, pssouza, francine\} @icmc.usp.br
}

\begin{abstract}
The teaching and learning of the subject memory hierarchy are not simple tasks, because many subjects that are covered in theory may demotivate learning because of its complexity. This paper presents the Learning Object Amnesia which aims to facilitate the construction of knowledge by simulating the structure and functionality of the memory hierarchy von Neumann architecture, specifically cache memory module, to allow analyze its performance. The experimental studies demonstrate an evolution in learning when this object was used, and in addition students' motivation to use other objects to assist in learning increased.
\end{abstract}

Resumo. $O$ ensino e a aprendizagem do tema hierarquia de memória não são tarefas simples, pois muitos assuntos que são abordados em teoria podem desmotivar a aprendizagem em virtude de sua complexidade. Este artigo apresenta o Objeto de Aprendizagem Amnesia, que visa facilitar a construção do conhecimento através da simulação da estrutura e da funcionalidade da hierarquia de memória na arquitetura von Neumann, mais especificamente o módulo de memória cache, para permitir analisar seu desempenho. Os estudos experimentais realizados demonstram uma evolução na aprendizagem quando se fez o uso deste objeto, além de se perceber que a motivação dos alunos em utilizar outros objetos de aprendizagem aumentou.

\section{Introdução}

A profissão de professor do ensino superior é um grande desafio. Ao mesmo tempo que se deve transmitir uma quantidade grande de informação para um aluno que já se encontra no nível superior, deve-se também atentar para o modo como esse conhecimento é transmitido. Um fator de desmotivação dos alunos que ingressam na universidade é a falta de conexão entre teoria e prática. Isso pode fazer com que os alunos abandonem os cursos, provocando evasão e um prejuízo para o país, dado que sabe-se que o mercado está carente de profissionais bem qualificados [BRASSCOM 2014].

Alguns recursos vêm sendo desenvolvidos para melhorar as formas de aprendizagem nas disciplinas dos cursos de tecnologia de informação e, dentre estes recursos, destacam-se os "Objetos de Aprendizagem" ou Learning Objects. De acordo com grupo Learning Objects Metadata Workgroup, objetos de aprendizagem podem ser definidos como: "Qualquer entidade, digital ou não digital, que possa ser utilizada, 
reutilizada ou referenciada durante o aprendizado suportado por tecnologias" [IEEE 2014]. Outra definição, menos abrangente, refere-se a objetos de aprendizagem como "Quaisquer recursos digitais que possam ser (re)utilizados como apoio ao ensino" [Wiley 2000].

Este artigo apresenta o projeto Amnesia, que desde 2012 vem transformando uma ferramenta, também chamada Amnesia, em um objeto de aprendizagem para apoiar o ensino e a aprendizagem da disciplina de Organização e Arquitetura de Computador. O objeto Amnesia tem por objetivo simular a estrutura, a funcionalidade e permitir analisar o desempenho da hierarquia de memória na arquitetura de von Neumann, considerando os registradores internos ao processador, as caches, a memória principal e o disco (considerando a técnica de paginação em memória virtual) [Oliveira, Santos, et al. 2008]. Este artigo aborda o módulo responsável pela memória cache no Amnesia.

Considerando especificamente a memória cache, a exposição prática deste conteúdo, com simulações dos conceitos apresentados na teoria, torna-se um fator positivo no processo de aprendizagem. Por meio da interação visual é possível que o aluno compreenda melhor o conteúdo, sem potencializar uma possível desmotivação imposta pelo método tradicional de ensino [Gadelha, Azevedo, et al. 2010].

Além disso, como o Amnesia depende da total interação do aluno para seu funcionamento, este último é beneficiado, pois estará, a todo o momento, completamente inserido no contexto de aprendizagem e participando da construção do seu próprio conhecimento de uma forma mais prática [Boff e Reategui 2005].

É importante ressaltar que o objeto de aprendizagem apresentado neste artigo não deverá substituir o professor. Muito pelo contrário, ele deverá ser utilizado como um apoio à aprendizagem visando tornar a participação do aluno mais efetiva na disciplina.

As demais seções deste artigo estão estruturadas da seguinte forma: a Seção 2 apresenta os trabalhos relacionados. A Seção 3 apresenta o objeto de aprendizagem Amnesia e o modo como foi utilizado nos cursos de Organização e Arquitetura de Computador do Laboratório de Sistemas Distribuídos e Programação Concorrente (LASDPC) do Instituto de Ciências Matemáticas e de Computação (ICMC/USP). A Seção 4 apresenta uma avaliação de efetividade da aprendizagem e uma avaliação do uso do objeto Amnesia. Por fim, as considerações finais e os trabalhos futuros são apresentados na Seção 5.

\section{Trabalhos Relacionados}

Alguns simuladores de hierarquia de memória, mais especificamente memória cache com diferentes níveis de complexidade e funcionalidade, foram comparados com o objeto Amnesia. Os simuladores analisados foram: Cache Memory Simulator [Chalk 2014], Didatic Cache Memory Simulator (DCMSim) [Cordeiro, Stefani, et al. 2003], SimpleScalar [Austin, Larson, et al. 2002] e Multitask Cache Simulator [Koren 2014].

O Cache Memory Simulator caracteriza-se como uma ferramenta gráfica que permite a interação do usuário por meio de controles predefinidos. Fornece estruturas de laço único, loop aninhado, entre outras, para serem executadas. Após a execução, é possível a análise dos dados por meio da observação do $\log$ e das taxas de acertos e falhas que também são providas pela ferramenta. Além disso, permite a configuração da 
memória cache com diferentes tamanhos, tamanhos de blocos e algoritmos de substituição [Chalk 2014].

O Didatic Cache Memory Simulator, mais conhecido como DCMSim, caracteriza-se como uma ferramenta gráfica que permite a simulação da memória cache. Possui uma interface amigável e atraente bem como todas as funcionalidades nela existentes e um painel que permite a visualização dos blocos contidos na memória principal. Os dados estatísticos são detalhados com tempos de acesso à memória cache e principal e um $\log$ de operações detalhado que permite a visualização de todas as posições que foram acessadas durante a execução de determinadas instruções. Quanto à configuração, permite o ajuste do tipo da memória cache em unificado ou separado e o ajuste da política de escrita em write-through ou write-back [Cordeiro, Stefani, et al. 2003].

O SimpleScalar caracteriza-se como uma ferramenta texto que permite a simulação da hierarquia de memória de um computador com memória cache unificada e separada. O log das instruções executadas, disponibilizado em arquivo texto, fornece os dados estatísticos com taxas de acertos, falhas e tempos de acesso. Um diferencial desta ferramenta consiste no apoio aos programas escritos na linguagem C [Austin, Larson, et al. 2002].

O Multitask Cache Simulator é uma ferramenta gráfica capaz de modelar uma cache de pequeno porte em um sistema que oferece apoio à multitarefa. Permite a configuração da cache com diferentes capacidades, algoritmos de substituição e tamanhos de blocos [Koren 2014].

O objeto de aprendizagem Amnesia, como as demais ferramentas analisadas, visam auxiliar o ensino da hierarquia de memória, simulando o uso de recursos como registradores, caches e memória. No entanto, o Amnesia tem um escopo abrangente, profundo e flexível, considerando aspectos funcionais, estruturais e de desempenho, desde registradores até memória secundária, diferenciando-o das demais ferramentas. Com uma interface textual e uma interface gráfica que possibilitam a simulação de um programa em um ambiente previamente configurado, permite a visualização em tempo real das informações contidas na memória principal e sua alocação detalhada na memória cache. O Amnesia integra também CPU, memória cache, memória principal e a paginação feita pela memória virtual, incluindo o uso de disco. Sua descrição completa, bem como seu funcionamento detalhado, será descrito na Seção 3 deste artigo. Outro diferencial do Amnesia baseia-se no seu objetivo de ser um objeto de aprendizagem, associado a planos de aula e tutoriais utilizados para aprimorar o nível de aprendizado dos alunos como será demonstrado na Seção 4 deste artigo.

\section{Objeto de Aprendizagem Amnesia}

O objeto de aprendizagem Amnesia caracteriza-se por ser um simulador de hierarquia de memória na arquitetura de von Neumann. Sua função é demonstrar o funcionamento dos registradores de um processador, memórias caches, principal e virtual de forma didática, com o objetivo de facilitar e melhorar a aprendizagem na disciplina de Organização e Arquitetura de Computadores. 


\subsection{Descrição do Objeto Amnesia}

Os principais conceitos abordados pelo Amnesia referem-se à disciplina de Organização e Arquitetura de Computadores, mais especificamente ao assunto hierarquia de memória, tais como: palavra, bloco, página, princípios de localidade temporal e espacial, caches multiníveis, acertos e falhas de cache, bem como algoritmos para tratamento das falhas, algoritmos de mapeamento direto e associativo (totalmente associativo ou por conjunto), transferência dos dados entre os níveis de memória, memória virtual, tabela de páginas e Translation Lookaside Buffer (TLB).

Esses conceitos são abordados pelo objeto durante a utilização de suas funcionalidades que se encontram distribuídas em três módulos:

- Módulo Processador: baseado na arquitetura MIPS, acrônimo de Microprocessor without Interlocked Pipeline Stages, proposta por Patterson e Henessy com o objetivo de simular o funcionamento de um processador RISC multiciclo de 32 bits bem como de seus registradores [Patterson e Hennessy 2005];

- Módulo Cache: responsável por simular memórias caches do tipo Unificada (dados e instruções em uma única cache) ou Separada (dados e instruções em caches separadas) bem como memórias caches multiníveis (L1, L2, L3, etc);

- Módulo Memória Virtual: responsável por simular endereços virtuais, memória associativa TLB e os acessos a disco em virtude de uma possível paginação;

As Figuras 1a e 1b ilustram o funcionamento geral do objeto Amnesia.
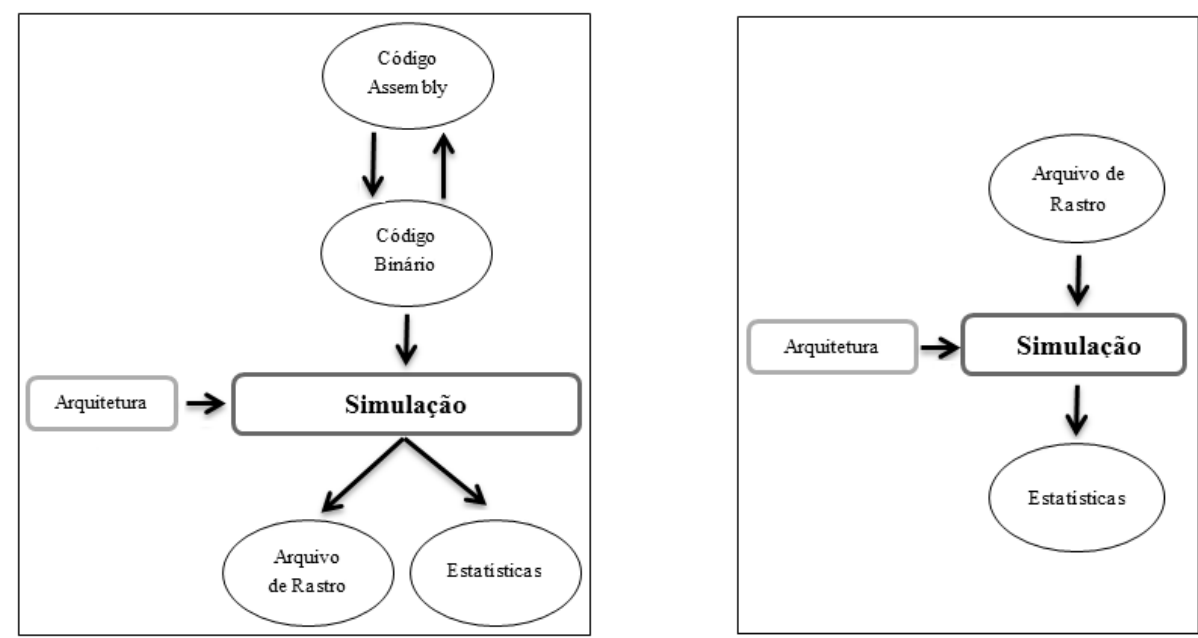

Figura 1. a) Funcionamento do Amnesia a partir de um código binário; b) Funcionamento do Amnesia a partir de um arquivo de rastro.

Para iniciar uma simulação é necessária a indicação de uma arquitetura mantida em um arquivo formatado no padrão $\mathrm{xml}$. Nesse arquivo, atributos de processador, memória cache, principal e virtual podem ser configurados. Para realizar uma simulação com o objetivo de analisar o funcionamento da memória cache, os atributos do processador, da memória cache e principal devem ser obrigatoriamente especificados. Desta forma, para o processador deve-se configurar o tamanho da palavra em bytes. Para cada nível da memória cache deve-se configurar o seu tipo (unificada ou separada), seu 
tamanho em palavras, o número de palavras por bloco, o número de blocos por conjunto (i.e. a associatividade), o número de ciclos para cada acesso de leitura, o número de ciclos para cada acesso de escrita, o tempo de cada ciclo, a política de substituição (FIFO ou LRU) e a política de atualização (write-through ou write-back). Para a memória principal deve-se configurar seu tamanho em palavras, o número de palavras por bloco, o número de ciclos para cada acesso de leitura, o número de ciclos para cada acesso de escrita e o tempo de cada ciclo.

Observando-se a Figura 1a, nota-se que a simulação pode ser conduzida a partir de uma sequência de códigos binários originados pela conversão de um código assembly MIPS, gerando estatísticas e um arquivo de rastro (trace) no padrão DIN [Hill 2014] contendo os acessos realizados na memória principal. Já a Figura 1b, mostra que uma simulação pode ser conduzida a partir de um arquivo de rastro (trace) no padrão DIN, gerando estatísticas.

Seja por código binário ou arquivo de rastro, a simulação pode ser executada de forma direta ou passo a passo. A Figura 2, que simula a execução passo a passo de um programa a partir de um arquivo de rastro, mostra que este tipo de execução provê um $\log$ detalhado e em tempo real da alocação das informações da memória principal na memória cache, possibilitando visualizar o espalhamento dos bits de um endereço requisitado e a determinação do byte-offset, word-offset, set e tag a cada instrução executada.

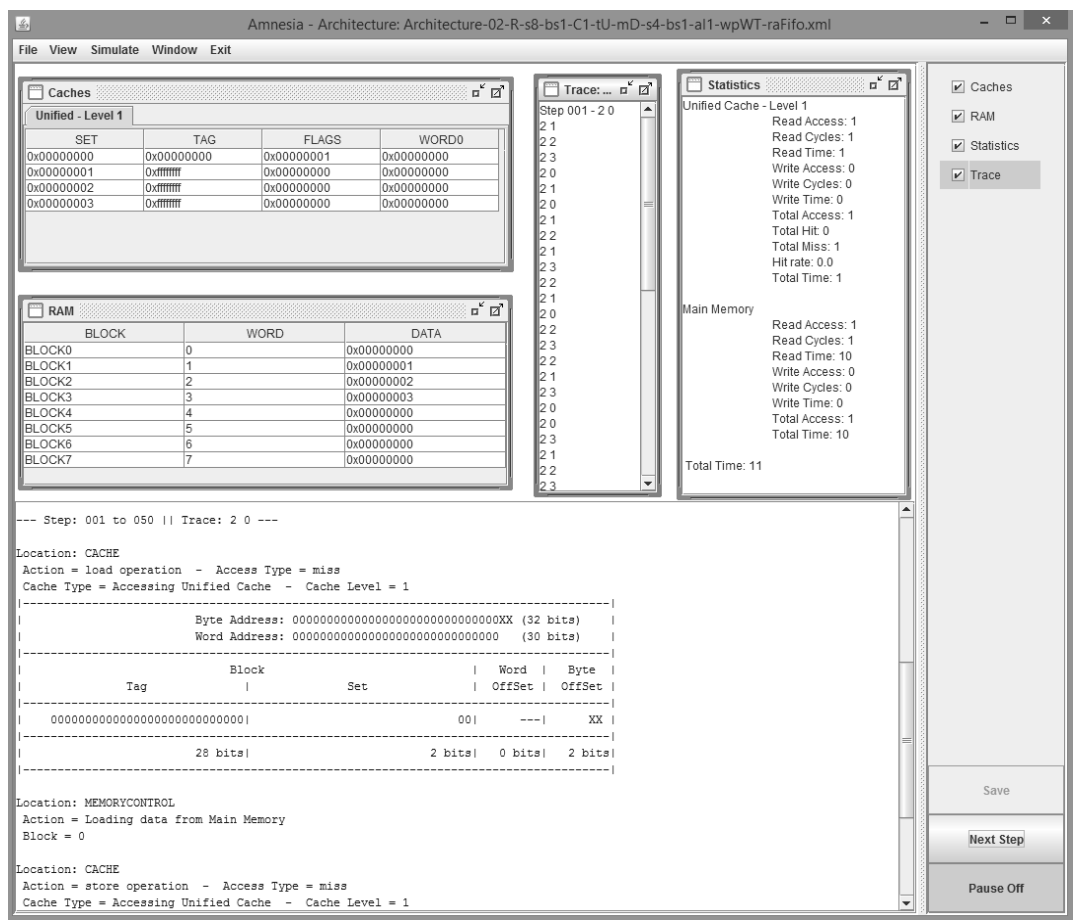

Figura 2. Execução passo a passo de um arquivo de rastro

Continuando a análise da Figura 2, também é possível visualizar o arquivo de rastro (trace) que é executado e o passo exato de execução, os dados contidos na memória cache e principal bem como suas estatísticas de acesso. Para cada nível de memória cache, pode-se analisar seus aspectos de desempenho a partir do número de acessos, número de ciclos e tempo de leitura, número de acessos, número de ciclos e 
tempo de escrita, número total de acessos, número total de acertos (hit) e de falhas (miss), percentual de acerto (hit rate) e o tempo total de execução. Já para a memória principal, pode-se analisar o número de acessos, número de ciclos e tempo de leitura, número de acessos, número de ciclos e tempo de escrita, número total de acessos e o tempo total de execução. Desta forma, é possível que o aluno verifique, de maneira clara, os aspectos de desempenho envolvendo a execução de um programa em um determinado ambiente.

Ressalta-se que um mesmo código binário ou arquivo de rastro pode ser executado em diferentes arquiteturas com configurações distintas de processador, memória cache, principal ou virtual. Essa característica é importante para que o aluno possa executar a mesma sequência de instruções em diferentes ambientes e comparar os resultados obtidos.

Após o término da simulação, um log pode ser gravado contendo as informações da arquitetura utilizada, código binário ou arquivo de rastro executado, espalhamento dos bits de cada endereço de memória requisitado e as estatísticas de acesso às memórias cache, principal e virtual.

\subsection{Utilização do Objeto Amnesia como Apoio ao Ensino}

Uma atividade prática, utilizando o Amnesia no apoio ao ensino, foi realizada para demonstrar e reforçar o conhecimento já adquirido do conteúdo de memória cache ministrado na disciplina de Organização e Arquitetura de Computador (ICMC/USP). Nesta atividade prática, com duração aproximada de 2 horas, foram abordados os seguintes assuntos: motivação e conceitos, funções de mapeamento, políticas de substituição e de atualização, aspectos de desempenho, caches multiníveis e caches unificadas e separadas.

Ao todo, foram realizados 11 exercícios com objetivos distintos para demonstrar a importância da memória cache a partir da exposição dos princípios de localidade temporal e espacial, tipos de acesso (acerto ou falha), funcionamento de cada tipo de mapeamento (direto, totalmente associativo e associativo por conjunto) bem como as políticas de substituição (FIFO e LRU), o espalhamento de bits (byte-offset, word-offset, set e tag) de um endereço de memória requisitado, operações de escrita e as políticas de atualização (write-through e write-back), caches multiníveis e o conceito da penalidade por falta e a diferença de caches separadas em relação às unificadas quanto à busca de instruções e dados.

Em todos os exercícios foram coletadas as estatísticas dos acessos com objetivo de analisar e comparar aspectos de desempenho como: taxas de acerto e falta e tempo total de execução, para que o aluno compreendesse e reafirmasse, na prática, o conceito visto na teoria em sala de aula.

A condução de cada exercício foi realizada por um monitor e acompanhada por cada aluno. O Amnesia foi instalado pelo próprio aluno em sua máquina local e todos os exercícios foram executados individualmente, dependendo da total interação deste aluno para sua conclusão, mantendo-o completamente inserido no contexto de aprendizagem e participando da construção do seu próprio conhecimento. 


\section{Avaliação do Objeto Amnesia}

Para avaliar a efetividade do objeto de aprendizagem Amnesia foi realizado um experimento com os alunos da disciplina de Organização e Arquitetura de Computador (ICMC/USP) que já conheciam todo o conteúdo de memória cache em virtude das aulas teóricas ministradas previamente em sala de aula pelos professores.

O experimento foi composto de dois testes distintos com níveis semelhantes de dificuldade, contendo 15 questões cada um e abordando todo o conteúdo teórico de memória cache visto em sala de aula. Também foi conduzida uma aula prática, utilizando o objeto de aprendizagem Amnesia abordando todos os assuntos relativos à memória cache conforme descrito na Seção 3.2.

Em síntese, os alunos deveriam responder às questões do primeiro teste (préteste) em aproximadamente 15 minutos baseando-se no conhecimento teórico obtido em sala de aula, depois deveriam participar da aula prática utilizando o objeto de aprendizagem Amnesia e por fim responder às questões do segundo teste (pós-teste) em aproximadamente 15 minutos com o conhecimento obtido em sala de aula, complementado pela utilização do Amnesia. Ressalta-se que um intervalo de 5 dias entre a aplicação do pré-teste e a realização da aula prática, também foi utilizado entre esta aula e a aplicação do pós-teste, com o objetivo de não favorecer possíveis resultados positivos.

Além da avaliação da efetividade de aprendizagem, neste experimento também foi avaliada a utilização do objeto Amnesia. No dia da realização da aula prática, foram respondidos dois questionários. O primeiro, de expectativa de uso, antes da utilização do Amnesia; o segundo, logo após o término da aula, tratava da reação ao uso.

\subsection{Avaliação da Efetividade de Aprendizagem}

A aplicação do pré-teste contou com a participação de 102 alunos, do pós-teste com 93 alunos e a aula prática com 32 alunos. Dentre todos os cenários obtidos no experimento, destacam-se dois: (i) cenário 1, composto por 23 alunos que participaram dos dois testes e da aula prática; e (ii) cenário 2, composto por 52 alunos que participaram dos dois testes e não participaram da aula prática.

Analisando a Figura 3 pode-se notar um melhor aproveitamento dos alunos do cenário 1 quanto à participação no pós-teste. Os alunos do cenário 1 acertaram 165 questões no pré-teste e 205 no pós-teste, demonstrando uma evolução de $24 \%$. Já os alunos do cenário 2, acertaram 443 questões no pré-teste e 476 no pós-teste, demonstrando um crescimento de $7 \%$. Desta forma, observa-se que a atividade prática com a utilização do objeto Amnesia, neste experimento, influenciou positivamente na sedimentação do conhecimento obtido por cada aluno.

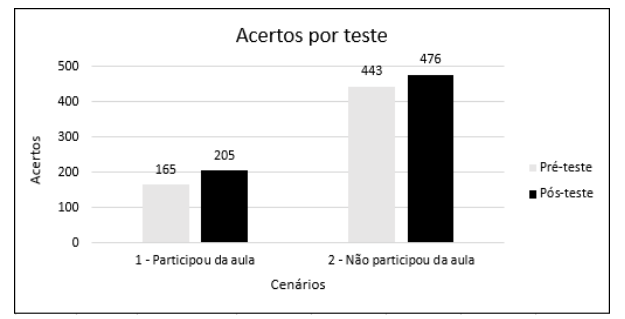

Figura 3. Gráfico de acertos por prova 
As Figuras 4 e 5 mostram o número de acertos por assunto nos cenários 1 e 2 respectivamente. Para a maioria dos assuntos pode-se notar uma evolução no conhecimento dos alunos do cenário 1, exceto para funções de mapeamento e política de substituição. Quando comparados estes dados com os do cenário 2, observa-se que dentre os assuntos que apresentaram queda de desempenho, também estão presentes funções de mapeamento e política de substituição. Esta informação é importante para que a queda do desempenho nestes assuntos não seja relacionada com a utilização do objeto Amnesia. Os fatores que possam justificar essa queda de desempenho nos dois assuntos e nos dois cenários ainda não foram investigados em profundidade e, portanto, não são conclusivos. Algumas possibilidades em investigação são: nível de dificuldade do pós-teste, dificuldade de entendimento do pós-teste, dificuldades pontuais nesses itens da matéria e falta de uma ênfase maior com o uso do objeto de aprendizagem nesses assuntos.

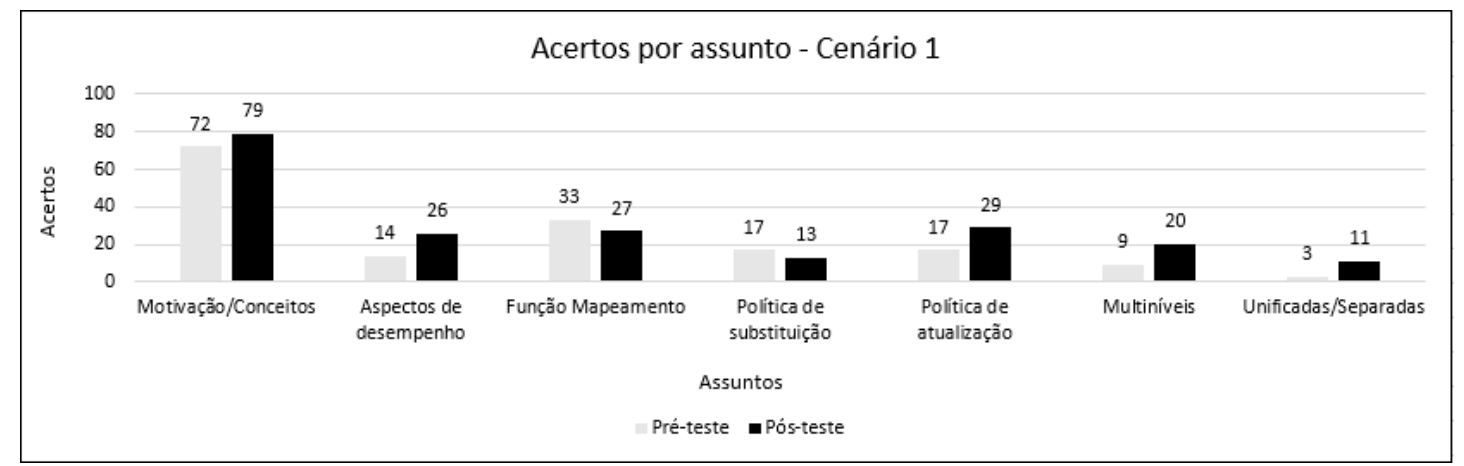

Figura 4. Gráfico de acertos por assunto referentes ao Cenário 1

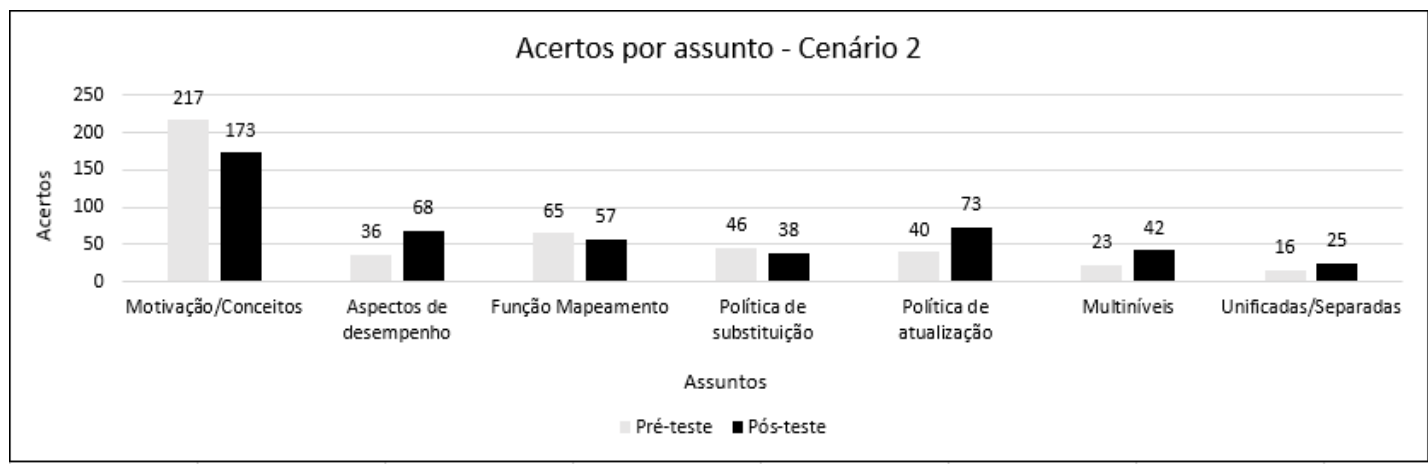

Figura 5. Gráfico de acertos por assunto referentes ao Cenário 2

Ainda comparando os cenários 1 e 2 a partir das Figuras 4 e 5, observa-se que os alunos que utilizaram o Amnesia evoluíram consideravelmente no desempenho das questões referentes à motivação/conceitos, caches multiníveis e unificadas/separadas. Como o aluno participou da construção do seu próprio conhecimento com o auxílio do Amnesia, notou-se uma maior preocupação quanto ao entendimento dos exercícios e os conceitos neles envolvidos, justificando o melhor desempenho em motivação/conceitos. Com relação aos assuntos sobre caches multiníveis e unificadas/separadas, sabe-se que eles demandam uma arquitetura de memória cache complexa para ser explicada na teoria e a utilização do objeto Amnesia facilitou a compreensão, devido à simulação prática. 


\subsection{Avaliação do Uso}

A avaliação do uso contou com a participação de 32 alunos. Observou-se que $37 \%$ dos alunos estavam em dúvida em relação a utilizar o Amnesia futuramente e 38,24\% possuíam um conhecimento regular sobre o conteúdo abordado antes de utilizar o objeto. Após a utilização, 97,06\% responderam que utilizariam o objeto novamente, $88,24 \%$ sentiram-se seguros quanto aos resultados nele obtidos, 58,82\% declararam saber o conteúdo um pouco mais do que antes e $29,41 \%$ saber muito mais. Esta última informação também pôde ser observada a partir da avaliação de aprendizagem.

Aliados a estes dados, $85,29 \%$ dos alunos consideraram que o conteúdo abordado na teoria em sala de aula tornou-se mais interessante com a utilização do objeto de aprendizagem Amnesia e 73,53\% consideraram que a motivação em utilizar outros objetos para auxiliar na aprendizagem aumentou, ressaltando a importância da construção do conhecimento sobre um determinado conteúdo ser obtido de uma forma mais prática.

\section{Considerações Finais e Trabalhos Futuros}

Este artigo apresentou o objeto de aprendizagem Amnesia, discutindo sua utilização como apoio ao ensino e à aprendizagem da disciplina de Organização e Arquitetura de Computador, mais especificamente o módulo de memória cache, visando melhorar a qualidade de ensino de hierarquia de memória.

De modo geral, foi possível observar que a exposição prática de determinados conteúdos, com simulações dos conceitos apresentados na teoria em sala de aula, aprimoraram o nível de aprendizagem dos alunos, conforme discutido na avaliação de aprendizagem descrita Seção 4.1.

Como o objeto Amnesia estimula a total interação do aluno, ele permitiu ao aluno a participação efetiva na disciplina. Além disso, manteve o aluno completamente inserido no contexto do aprendizado e participativo na construção do seu próprio conhecimento, despertando uma maior preocupação quanto ao entendimento dos exercícios bem como os conceitos envolvidos. Essa conclusão pode ser justificada pela melhoria de desempenho em $24 \%$ dos alunos que utilizaram o Amnesia em relação aos $7 \%$ dos alunos que não o utilizaram.

Além disso, a partir da avaliação do uso do objeto Amnesia, descrita na Seção 4.2, pode-se perceber que a motivação dos alunos em utilizar outros objetos para auxiliar na aprendizagem aumentou, ressaltando a importância da construção do conhecimento sobre um conteúdo ser obtido de uma forma mais prática.

Para trabalhos futuros, novos experimentos estão sendo realizados com o objetivo de melhorar e aperfeiçoar os métodos de avaliação bem como a utilização do objeto de aprendizagem no apoio ao ensino. Junto a isso, novas implementações serão desenvolvidas, testadas e implantadas no objeto para facilitar, cada vez mais, sua utilização pelos alunos.

Por fim, o objeto de aprendizagem Amnesia será armazenado em um Repositório de Objetos de Aprendizagem (ROA) com o objetivo de disponibilizá-lo de forma segura e confiável através de uma plataforma distribuída para a manutenção e preservação do acervo digital [Kemczinski, Hounsell, et al. 2011]. 


\section{Agradecimentos}

Agradecimentos às agências de fomento FAPESP, CAPES e CNPq pelo apoio financeiro a este trabalho.

\section{Referências}

Austin, T., Larson, E., Ernst, D. (2002) "SimpleScalar: An Infrastructure for Computer System Modeling", Em: IEEE Computer Society, v.35, p.59-67.

Boff, E., Reategui, E. (2005) "A importância do processo de avaliação de software educativo.", Em: Anais do Seminário Nacional de Tecnologia na Educação, Caxias do Sul.

BRASSCOM. (2014) "Procuram-se profissionais de TI", Em: http://www.brasscom.org.br/brasscom/Portugues/detNoticia.php?codArea=2\&codCat egoria $=26 \& \operatorname{codNoticia}=400$, Maio.

Chalk, B. (2014) "Cache Memory Simulator", Em: http://myweb.lsbu.ac.uk/ chalkbs/research/CacheApplet.htm, Julho.

Cordeiro, E. S., Stefani, I. G. A., Soares, T. C. A. P., Martins, C. A. P. S. (2003) "DCMSim: Didatic Cache Memory Simulator", Em: IEEE Computer Society, v.2, p. F1C 14-19.

Gadelha, R. N. S., Azevedo, R. R., Oliveira, H. T. A. (2010) “OS Simulator: Um simulador de sistemas de arquivos para apoiar o ensino/aprendizagem de sistemas operacionais", Em: Anais do XXI Simpósio Brasileiro de Informática na Educação, João Pessoa.

Hill, M. (2014) "Dinero - cache simulator, version III", Em: http://www.ece.cmu.edu/ ece548/tools/dinero/man/dinero.htm, Julho.

IEEE LTSC WG12 (2014) "Learning Object Metadata", Em: http://ltsc.ieee.org/wg12/, Julho.

Kemczinski A., Hounsell M. S., Gasparini I., Filho R. G., Silva T. C. (2011) "Repositório de Objetos de Aprendizagem para a Área de Computação e Informática - ROAI", Em: Anais do XXII Simpósio Brasileiro de Informática na Educação, p. 234-243, Aracaju.

Koren, I. (2014) "Multitask Cache Simulator", Em: http://www.ecs.umass.edu/ece/koren/architecture/CacheTr/cacheSim.html, Julho.

Oliveira B. H., Santos J. H., Souza, P. S. L., Bruschi, S. M., Souza, S. R. S. (2008) "Amnesia: Um simulador de Hierarquia de Memória", Em: Anais do III Workshop sobre Educação em Arquitetura de Computadores, p. 13-16, Campo Grande.

Patterson, D. A. e Hennessy, J. L. (2005), Organização e projeto de computadores: a interface hardware/software, $3^{\text {a }}$ Edição.

Wiley, D. A. (2000) "Connecting learning objects to instructional design theory: A definition, a metaphor, and a taxonomy", Em: D. A. Wiley (Ed.), The Instructional Use of Learning Objects: Online Version, http://reusability.org/read/chapters/ wiley.doc, Junho. 